\title{
Composição florística do componente arbóreo de um trecho de Floresta Atlântica na Área de Proteção Ambiental da Serra da Capoeira Grande, Rio de Janeiro, RJ, Brasil ${ }^{1}$
}

\author{
Gustavo Luna Peixoto ${ }^{2,4}$, Sebastião Venâncio Martins², Alexandre Francisco da Silva ${ }^{3}$ e Elias Silva ${ }^{2}$
}

Recebido em 29/11/2002. Aceito em 29/07/2003

\begin{abstract}
RESUMO - (Composição florística do componente arbóreo de um trecho de Floresta Atlântica na Área de Proteção Ambiental da Serra da Capoeira Grande, Rio de Janeiro, RJ, Brasil). A Área de Proteção Ambiental (APA) da Serra da Capoeira Grande (2259’03”S e 4338'59'W) tem área total de 80 ha e é um dos últimos remanescentes florestais com pau-brasil (Caesalpinia echinata Lam.) no município do Rio de Janeiro. Além disso, ocorrem na área outras três espécies ameaçadas de extinção: Cariniana ianeirensis R. Knuth, Acosmium lentiscifolium Spreng. e Machaerium incorruptible (Vell.) Fr. All. ex Benth. O levantamento fitossociológico foi realizado por meio do método dos quadrantes, tendo sido alocados 200 pontos e tendo-se como critério de inclusão $15 \mathrm{~cm}$ de circunferência do tronco a $1,30 \mathrm{~m}$ de altura do solo. A composição florística é o resultado desta amostragem acrescida de coletas feitas durante caminhadas no fragmento, totalizando 29 famílias, 58 gêneros e 69 espécies. As famílias que apresentaram maior número de espécies foram: Leguminosae (13), Myrtaceae (6), Euphorbiaceae (5), Bignoniaceae, Bombacaceae, Celastraceae, Flacourtiaceae, Moraceae, Rubiaceae e Solanaceae (3). Analisando a similaridade florística entre a APA da Serra da Capoeira Grande e outras 18 áreas florestais do Rio de Janeiro, observou-se maior identidade florística entre a área estudada e florestas de baixada localizadas próximas ao mar. Todas as florestas reuniram-se com um baixo nível de similaridade, refletindo a diversidade florística das florestas do Rio de Janeiro.
\end{abstract}

Palavras-chave: florística, Floresta Atlântica, fragmentos florestais, conservação

\begin{abstract}
Floristic survey of the tree layer in an area of Atlantic Rainforest in Serra da Capoeira Grande Environmental Protection Area, Rio de Janeiro State, Brazil). The 80-hectare site (22 $59^{\prime} 03^{\prime \prime S}$ and $\left.43^{\circ} 38^{\prime} 59^{\prime \prime} \mathrm{W}\right)$, is one of the last forest remnants in Rio de Janeiro municipality, where brazilwood (Caesalpinia echinata Lam.) occurs naturally. Furthermore, three other endangered species occur in this area: Cariniana ianeirensis R. Knuth, Acosmium lentiscifolium Spreng., and Machaerium incorruptible (Vell.) Fr. All. ex Benth. Trees were sampled according to the point-centered-quarter method. The inclusion criteria was $\mathrm{PBH} \geq 15 \mathrm{~cm}$; a total of 200 points were surveyed. The floristic composition was comprised of the sampled individuals plus species collected during random forays inside the forest. A total of 69 species were obtained, in 58 genera and 29 families. The most species-rich families were Leguminosae (13), Myrtaceae (6), Euphorbiaceae (5) and Bignoniaceae, Bombacaceae, Celastraceae, Flacourtiaceae, Moraceae, Rubiaceae and Solanaceae (3). The floristic composition of Serra da Capoeira Grande Environmental Protection Area was compared to 18 other forest areas in Rio de Janeiro State. Greater floristic similarity was observed between this forest and those near the sea at lower altitudes. Similarity was low for all forests, showing the high biological diversity of Rio de Janeiro forests.
\end{abstract}

Key words: floristics, Atlantic forest, forest fragments, conservation

\section{Introdução}

A Floresta Atlântica é um bioma de grande complexidade biológica e foi considerado, pela União Internacional para Conservação de Natureza, como um dos mais ameaçados do mundo (IUCN 1986). Antes da colonização, este bioma estendia-se em faixa praticamente contínua, desde o Rio Grande do Norte até o Rio Grande do Sul, acompanhando o litoral e ocupando cerca de $12 \%$ do território nacional. Cinco séculos depois, a ocupação territorial reduziu a Floresta Atlântica a fragmentos florestais de variados tamanhos, restando hoje apenas 5\% de sua cobertura original (Consórcio Mata Atlântica 1992). No Estado do Rio de Janeiro, a floresta ombrófila densa ocupava, em 1994, $16,6 \%$ do território (CIDE 2000).

A cidade do Rio de Janeiro foi capital do país entre 1763 e 1960, atraindo grande contingente de imigrantes em busca de melhores condições de vida. Porém, a ocupação do território sempre se defrontou com

\footnotetext{
Parte da Tese de Mestrado do primeiro Autor

2 Departamento de Engenharia Florestal, Universidade Federal de Viçosa, CEP 36571-000, Viçosa, MG, Brasil

3 Departamento de Biologia Vegetal, Universidade Federal de Viçosa, CEP 36571-000, Viçosa, MG, Brasil

4 Autor para correspondência: glpeixoto@terra.com.br
} 
características naturais muito peculiares, gerando prejuízos sociais e ambientais de grande importância (Santana 2000). A expansão da cidade reduziu continuamente o componente florestal. As florestas foram removidas, primeiramente, nas baixadas, para a abertura de áreas para a agricultura e habitação, e, posteriormente, as áreas de encosta foram também utilizadas para fins agrícolas, principalmente no Ciclo do Café, nos séculos XVIII e XIX (Dean 1995). A fragmentação florestal, causada pela expansão humana nesta cidade, causou o isolamento de trechos florestais, impedindo o fluxo gênico e diminuindo a diversidade biológica.

Estudos sobre a composição da vegetação nos grandes maciços florestais do município têm sido feitos (Oliveira et al. 1995; Santana 2000), porém os pequenos fragmentos, muitos deles protegidos por unidades de conservação, não têm recebido a importância devida, o que tem permitido sua devastação. Neste contexto, este trabalho apresenta a composição florística do componente arbóreo de um trecho de Floresta Atlântica localizado no município de Rio de Janeiro e o compara com outras matas do Estado do Rio de Janeiro, visando fornecer subsídios para a sua conservação e das espécies que nele ocorrem.

\section{Material e métodos}

Área de estudo - A Área de Proteção Ambiental (APA) da Serra da Capoeira Grande (22 59'03"S e 4338'59"W) localiza-se em Pedra de Guaratiba, zona oeste do município do Rio de Janeiro e, embora pertença ao Complexo Geológico do Maciço da Pedra Branca, não se insere nos limites do Parque Estadual da Pedra Branca. Essa serra se destaca na planície da região de Guaratiba por alcançar altitude de até $159 \mathrm{~m}$ sustentando importante remanescente da mata de planície litorânea (Peixoto 1992) outrora abundante no município e hoje escassamente representada. $\mathrm{O}$ solo muito raso e rochoso na porção médio-superior da serra cria ambiente com limitações em oferta hídrica e de profundidade, restringindo maior desenvolvimento das árvores.

Clima - a região caracteriza-se por apresentar temperatura do ar média anual de $23,6^{\circ} \mathrm{C}$, sendo o mês de fevereiro o mais quente, com temperatura média de $26,7^{\circ} \mathrm{C}$, e junho, o mais frio, com média de $21,0^{\circ} \mathrm{C}$. O total médio anual de precipitação é de $1.027,2 \mathrm{~mm}$, sendo o mês de agosto o mais seco, com 47,4mm e março, o mais chuvoso, com 140,6mm (Menezes \& Araujo 1999). De acordo com o sistema de Köppen, o clima da região é do tipo Aw - Clima Tropical Chuvoso. As chuvas são abundantes no verão e escassas no inverno. Porém, apesar da região estar sujeita a certa estacionalidade, o diagrama ombrométrico mostrou a não ocorrência de mês seco durante o ano.

Análise do solo - para caracterização do solo, foram utilizadas três amostras compostas, cada uma por dez amostras simples, na profundidade de 0 a $20 \mathrm{~cm}$, na baixada, encosta e topo da serra. As análises foram realizadas pelo Departamento de Solos da Universidade Federal de Viçosa, utilizando-se a metodologia adotada pela Empresa Brasileira de Pesquisa Agropecuária (EMBRAPA 1979).

Levantamento florístico - Durante o período de dezembro/2000 a março/2002 foram feitas excursões quinzenais à APA. O levantamento florístico foi realizado por meio do método de quadrantes (Cottam \& Curtis 1956), com as modificações sugeridas por Martins (1993), tendo-se como critério de inclusão a circunferência do tronco a $1,30 \mathrm{~m}$ de altura do solo igual ou maior a $15 \mathrm{~cm}$. Foram alocados 200 pontos amostrais (de nove em nove metros), em linhas paralelas, distantes entre si de $10 \mathrm{~m}$. A composição florística é o resultado dessa amostragem, acrescida de outras coletas realizadas em caminhadas no fragmento. $\mathrm{O}$ material botânico foi depositado no Herbário do Instituto de Pesquisas Jardim Botânico do Rio de Janeiro (RB). A identificação foi realizada por comparação com exsicatas do citado herbário e consultas a especialistas, quando necessário, e os nomes populares foram obtidos em bibliografia.

Classificação sucessional das espécies - As espécies foram agrupadas em categorias sucessionais: pioneiras, secundárias iniciais e secundárias tardias, segundo a classificação proposta por Gandolfi et al. (1995) e utilizando como base os trabalhos de Gandolfi et al. (1995), Lorenzi (1998; 2000), Oliveira (1999), Santana (2000) e Martins et al. (2002). Para as espécies não encontradas na literatura, a classificação baseou-se em observações de campo sobre sua ocorrência em diferentes ambientes na floresta estudada e em outras áreas.

Comparação florística entre trechos de Floresta Atlântica - Para a comparação florística entre a área estudada e outros remanescentes de Floresta Atlântica do Estado do Rio de Janeiro, foram reunidos 19 trabalhos de florística e fitossociologia incluindo este. A partir de uma matriz de dados binários (presença/ausência) das espécies nas 19 áreas, produziu-se uma matriz de coeficientes de similaridade de Jaccard (Valentin 2000). 
Para interpretar as similaridade florística entre as áreas, empregou-se uma análise de agrupamento, pelo método média de grupo (UPGMA) (Valentin 2000). Todas as análises foram realizadas com o programa FITOPAC (Shepherd 1996). A validação dos nomes das espécies e a exclusão das sinonímias botânicas foi feita utilizando a base de dados fornecida pelo Missouri Botanical Garden (www.mobot.org, consulta realizada em 17/05/2002).

\section{Resultados e discussão}

Análise do solo - Para a maioria dos parâmetros avaliados, a amostra retirada da parte mais baixa do fragmento (Baixada) apresentou resultados melhores do que as retiradas na encosta e no topo da serra (Tab. 1). Isso ocorreu para o teor de cálcio e de magnésio, para a soma de bases trocáveis e para a saturação por bases. A acidez trocável $\left(\mathrm{Al}^{+++}\right)$e a acidez potencial $(\mathrm{H}+\mathrm{Al})$ mostraram também a melhor qualidade do solo da baixada, para o qual os valores destes parâmetros foram baixos; já para as outras duas amostras, o valor pode ser considerado alto. A saturação por alumínio para a baixada pode ser considerada muito baixa, enquanto que para a encosta e para o topo este valor pode ser considerado médio (Alvarez et al. 1999). Por outro lado, os teores de matéria orgânica apresentados pelos solos da baixada e da encosta podem ser considerados baixos, já valor apresentado pela amostra retirada do topo da serra é maior.

Tabela 1. Resultado das análises químicas do solo do trecho estudado na Área de Proteção Ambiental da Serra da Capoeira Grande, Rio de Janeiro, RJ. ( $\mathrm{SB}=$ soma de bases trocáveis; $\mathrm{t}=$ capacidade de troca catiônica efetiva; $\mathrm{T}=$ capacidade de troca catiônica em $\mathrm{pH}$ 7; $\mathrm{V}=$ saturação por bases; $\mathrm{m}$ = saturação por Alumínio; e $\mathrm{MO}=$ teor de matéria orgânica).

\begin{tabular}{lccc}
\hline Parâmetro & Baixada & Encosta & Topo \\
\hline $\mathrm{P}\left(\mathrm{mg} / \mathrm{dm}^{3}\right)$ & 2,49 & 3,39 & 3,50 \\
$\mathrm{P}-\mathrm{rem}(\mathrm{mg} / \mathrm{l})$ & 43,20 & 19,40 & 17,70 \\
$\mathrm{~K}\left(\mathrm{mg} / \mathrm{dm}^{3}\right)$ & 87,00 & 70,00 & 85,00 \\
$\mathrm{Ca}^{++}\left(\mathrm{cmol}_{\mathrm{c}} / \mathrm{dm}^{3}\right)$ & 1,52 & 0,80 & 0,86 \\
$\mathrm{Mg}^{++}\left(\mathrm{cmol}_{\mathrm{c}} / \mathrm{dm}^{3}\right)$ & 1,51 & 0,79 & 0,72 \\
$\mathrm{Al}++\left(\mathrm{cmol}_{\mathrm{c}} / \mathrm{dm}^{3}\right)$ & 0,24 & 1,68 & 1,56 \\
$\mathrm{H}+\mathrm{Al}\left(\mathrm{cmol}_{\mathrm{c}} / \mathrm{dm}^{3}\right)$ & 3,83 & 7,79 & 8,38 \\
$\mathrm{SB}\left(\mathrm{cmol} / \mathrm{dm}^{3}\right)$ & 3,25 & 1,77 & 1,80 \\
$\mathrm{t}\left(\mathrm{cmol} / \mathrm{dm}^{3}\right)$ & 3,49 & 3,45 & 3,36 \\
$\mathrm{~T}\left(\mathrm{cmol} / \mathrm{dm}^{3}\right)$ & 7,08 & 9,56 & 10,18 \\
$\mathrm{~V}(\%)$ & 45,90 & 18,50 & 17,00 \\
$\mathrm{~m}(\%)$ & 6,90 & 48,70 & 46,40 \\
$\mathrm{MO}(\mathrm{dag} / \mathrm{kg})$ & 2,02 & 3,43 & 4,43 \\
$\mathrm{pH}\left(\mathrm{H}_{2} \mathrm{O}\right)$ & 5,20 & 4,56 & 4,58 \\
\hline
\end{tabular}

$\mathrm{O} \mathrm{pH}\left(\mathrm{em} \mathrm{H}_{2} \mathrm{O}\right)$ em todas as amostras pode ser considerado baixo, segundo Alvarez et al. (1999). Além disso, para todas as amostras, o fósforo disponível é muito baixo. Apesar da variação na fertilidade do solo entre as posições topográficas, todas caracterizam-se por apresentarem solos distróficos. A posição topográfica de baixada exibiu condições de fertilidade pouco melhores que as demais, o que já era esperado, tendo em vista que esses locais recebem nutrientes carreados das partes mais altas do terreno.

Florística - Considerando os indivíduos amostrados nos quadrantes e os coletados fora da amostra, foram encontradas 69 espécies, pertencentes a 58 gêneros e 29 famílias. Duas morfo-espécies foram identificadas apenas ao nível genérico e duas ao nível de família. A Tabela 2 lista as espécies encontradas, bem como seus nomes populares e o grupo ecológico.

As famílias que apresentaram maior número de espécies foram Leguminosae, com 13 espécies (Mimosoideae com sete, Papilionoideae com cinco e Caesalpinioideae com uma), Myrtaceae, com seis, Euphorbiaceae representada por cinco espécies e Bignoniaceae, Bombacaceae, Celastraceae, Flacourtiaceae, Moraceae, Rubiaceae e Solanaceae, representadas por três espécies cada uma.

Comparando-se as famílias com maior número de espécies neste estudo e em outras áreas de Floresta Atlântica do Estado do Rio de Janeiro (Tab. 3), verifica-se que, apesar de Rodrigues (1996) e Kurtz \& Araújo (2000) também terem utilizado o método dos quadrantes, houve maior semelhança entre as florestas da Serra da Capoeira Grande, de Búzios (Farág 1999) e da Mata do Carvão, em São Francisco de Itabapoana (Silva \& Nascimento 2001), todas localizadas próximas ao litoral e em altitudes menores. No conjunto das cinco áreas comparadas, Myrtaceae ocupou a primeira ou segunda posição em riqueza, Leguminosae aparece em destaque em quatro áreas, e Euphorbiaceae, em três.

Leguminosae e Myrtaceae são as famílias que apresentam maior riqueza específica na maioria dos levantamentos realizados no Domínio da Mata Atlântica (Mori et al. 1983; Peixoto \& Gentry 1990; Silva \& Nascimento 2001). Lauraceae e Rubiaceae que, de modo geral, estão incluídas em diversos estudos como famílias mais ricas em espécies (Rodrigues 1996; Guedes-Bruni et al. 1997), estão representadas neste fragmento por apenas uma e três espécies, respectivamente.

Segundo Leitão-Filho (1993) e Tabarelli (1997), Myrtaceae e Lauraceae são características de florestas 
154 Peixoto, Martins, Silva \& Silva: Composição florística do componente arbóreo de um trecho de Floresta Atlântica...

Tabela 2. Lista das espécies amostradas na Área de Proteção Ambiental da Serra da Capoeira Grande, Rio de Janeiro, respectivos nomes populares e grupos ecológicos sucessionais. $(\mathrm{SI}=$ secundária inicial; $\mathrm{ST}=$ secundária tardia; $\mathrm{SC}=$ sem classificação; $\mathrm{P}=$ pioneira).

\begin{tabular}{|c|c|c|}
\hline Famílias/Espécies & Nomes populares & Grupo ecoló \\
\hline \multicolumn{3}{|l|}{ ANACARDIACEAE } \\
\hline Astronium fraxinifolium Schott ex Spreng. & gonçalo-alves & SI \\
\hline Mangifera indica $\mathrm{L}$. & mangueira & $\mathrm{SC}$ \\
\hline Spondias lutea $\mathrm{L}$. & caja-mirim & ST \\
\hline \multicolumn{3}{|l|}{ APOCYNACEAE } \\
\hline Aspidosperma subincanum Mart. & - & ST \\
\hline Peschiera laeta (Mart.) Miers & - & $\mathrm{SC}$ \\
\hline \multicolumn{3}{|l|}{ BIGNONIACEAE } \\
\hline Jacaranda macrantha Cham. & carobão & SI \\
\hline Sparattosperma leucanthum (Vell.) K. Schum. & ipê-cinco-chagas & $\mathrm{P}$ \\
\hline Tabebuia impetiginosa (Mart. ex DC.) Standl. & ipê-amarelo & $\mathrm{SC}$ \\
\hline \multicolumn{3}{|l|}{ BOMBACACEAE } \\
\hline Bombacopsis glabra (Pasq.) A. Robyns & - & SI \\
\hline Chorisia speciosa A. St.-Hil. & painera & SI \\
\hline Pseudobombax grandiflorum (Cav.) A. Robyns & imbiruçú & $\mathrm{P}$ \\
\hline \multicolumn{3}{|l|}{ CAPPARACEAE } \\
\hline Capparis flexuosa (L.) L. & - & $\mathrm{P}$ \\
\hline \multicolumn{3}{|l|}{ CELASTRACEAE } \\
\hline Maytenus communis Reissek & - & ST \\
\hline Maytenus ilicifolia (Burch. ex London) Planch. & espinheira-santa & ST \\
\hline Maytenus robusta Reissek & coração-de-bugre & ST \\
\hline \multicolumn{3}{|l|}{ ERYTHROXYLACEAE } \\
\hline Erythroxylum cuspidifolium Mart. & caga-fede & ST \\
\hline Erythroxylum pulchrum A. St.- Hil. & caga-fede & SI \\
\hline \multicolumn{3}{|l|}{ EUPHORBIACEAE } \\
\hline Actinostemon communis (Müll. Arg.) Pax & laranjeira-brava & ST \\
\hline Bernardia axillaris Müll. Arg. & - & $\mathrm{SC}$ \\
\hline Margaritaria nobilis L.f. & figueirinha & ST \\
\hline Sebastiania multiramea Müll. Arg. & - & ST \\
\hline Sebastiania serrata (Baill. ex Müll. Arg.) Müll. Arg. & branquilho & ST \\
\hline \multicolumn{3}{|l|}{ FLACOURTIACEAE } \\
\hline Casearia commersoniana Cambess. & - & ST \\
\hline Casearia obliqua Spreng. & - & $\mathrm{SC}$ \\
\hline Casearia sylvestris $\mathrm{Sw}$. & guaçatonga & $\mathrm{P}$ \\
\hline \multicolumn{3}{|l|}{ LAURACEAE } \\
\hline Ocotea divaricata (Nees) Mez & canela & ST \\
\hline \multicolumn{3}{|l|}{ LECYTHIDACEAE } \\
\hline Cariniana ianeirensis R. Knuth & jacarandá & ST \\
\hline \multicolumn{3}{|l|}{ LEGUMINOSAE/CAESALPINIOIDEAE } \\
\hline Caesalpinia echinata Lam. & pau-brasil & ST \\
\hline \multicolumn{3}{|l|}{ LEGUMINOSAE/MIMOSOIDEAE } \\
\hline Albizia polycephala (Benth.) Killip & monjolo-alho & SI \\
\hline Anadenanthera colubrina (Vell.) Brenan & angico-branco & SI \\
\hline Inga lentiscifolia Benth. & ingá & SI \\
\hline Inga subnuda Salzm. ex Benth. ssp. luschnathiana (Benth.) T.D. Penn. & ingá & SI \\
\hline Piptadenia gonoacantha (Mart.) J.F. Macbr. & pau-jacaré & SI \\
\hline Piptadenia paniculata Benth. & cangiquinha & SI \\
\hline Pseudopiptadenia contorta (DC.) G.P. Lewis \& M.P. Lima & monjolo-sabão & SI \\
\hline \multicolumn{3}{|l|}{ LEGUMINOSAE/PAPILIONOIDEAE } \\
\hline Acosmium lentiscifolium Schott & moço-branco & $\mathrm{SC}$ \\
\hline Machaerium aculeatum (Vell.) Stellfeld & bico-de-pato & SI \\
\hline Machaerium incorruptible (Vell.) Fr. All. ex Benth. & jacarandá-cipó & $\mathrm{P}$ \\
\hline Pterocarpus rohrii Vahl & pau-sangue & SI \\
\hline Swartzia myrtifolia J.E. Smith & - & SI \\
\hline
\end{tabular}


Tabela 2 (continuação)

\begin{tabular}{|c|c|c|}
\hline Famílias/Espécies & Nomes populares & Grupo ecológico \\
\hline \multicolumn{3}{|l|}{ LOGANIACEAE } \\
\hline Strychnos atlantica Krukoff \& Barneby & - & $\mathrm{SC}$ \\
\hline \multicolumn{3}{|l|}{ MELASTOMATACEAE } \\
\hline Miconia jucunda (DC.) Triana & - & $\mathrm{SC}$ \\
\hline \multicolumn{3}{|l|}{ MORACEAE } \\
\hline Cecropia lyratiloba Miq. & embaúba & $\mathrm{P}$ \\
\hline Ficus luschnathiana (Miq.) Miq. & pau-de-leite & SI \\
\hline Sorocea bonplandii (Baill.) Burger, Lanj. \& Boer & folha-de-serra & ST \\
\hline \multicolumn{3}{|l|}{ MYRSINACEAE } \\
\hline Myrsine umbellata Mart. & capororoca-branca & SI \\
\hline \multicolumn{3}{|l|}{ MYRTACEAE } \\
\hline Campomanesia eugenioides (Cambess.) D. Legrand & guabiroba & $\mathrm{ST}$ \\
\hline Eugenia maroviana $\mathrm{O}$. Berg & - & \\
\hline Psidium guajava $\mathrm{L}$. & goibeira & $\mathrm{P}$ \\
\hline Syzygium cumini (L.) Skeels & jamelão & \\
\hline Myrtaceae sp. 1 & - & $\mathrm{SC}$ \\
\hline Myrtaceae sp. 2 & - & ST \\
\hline \multicolumn{3}{|l|}{ NYCTAGINACEAE } \\
\hline Guapira hirsuta (Choisy) Lundell & maria-mole & $\mathrm{ST}$ \\
\hline Guapira opposita (Vell.) Reitz & maria-mole & ST \\
\hline \multicolumn{3}{|l|}{ OCHNACEAE } \\
\hline Ouratea stipulata (Vell.) Sastre & - & $\mathrm{SC}$ \\
\hline \multicolumn{3}{|l|}{ PALMAE } \\
\hline Syagrus romanzoffiana (Cham.) Glassm. & jerivá & SI \\
\hline \multicolumn{3}{|l|}{ PHYTOLACACEAE } \\
\hline Seguieria floribunda Benth. & limoeiro-bravo & SI \\
\hline \multicolumn{3}{|l|}{ POLYGONACEAE } \\
\hline Ruprechtia lundii Meisn. & - & SI \\
\hline \multicolumn{3}{|l|}{ RUBIACEAE } \\
\hline Alseis involuta K. Schum. & - & ST \\
\hline Coutarea hexandra (Jack.) K. Schum. & - & ST \\
\hline Psychotria carthagenensis Jacq. & - & $\mathrm{ST}$ \\
\hline \multicolumn{3}{|l|}{ RUTACEAE } \\
\hline Pilocarpus spicatus A. St.-Hil. & - & $\mathrm{SC}$ \\
\hline \multicolumn{3}{|l|}{ SAPINDACEAE } \\
\hline Cupania vernalis Cambess. & camboatá & SI \\
\hline \multicolumn{3}{|l|}{ SOLANACEAE } \\
\hline Metternichia princeps Mik. & jasmim-do-morro & SI \\
\hline Solanum swartzianum Roem. \& Schult. & - & $\mathrm{P}$ \\
\hline Solanum sp. & - & $\mathrm{P}$ \\
\hline \multicolumn{3}{|l|}{ TILIACEAE } \\
\hline Luehea candicans Mart. & - & SI \\
\hline \multicolumn{3}{|l|}{ ULMACEAE } \\
\hline Celtis iguanea (Jack.) Sarg. & - & $\mathrm{P}$ \\
\hline \multicolumn{3}{|l|}{ VIOLACEAE } \\
\hline Rinorea laevigata (Sol. ex Ging.) Hekking & - & $\mathrm{SC}$ \\
\hline
\end{tabular}

mais maduras. Portanto, apesar do destaque de Myrtaceae em riqueza específica, a baixa representatividade de Lauraceae e Rubiaceae, ambas com muitas espécies tardias, pode sugerir que a floresta da Serra da Capoeira Grande se encontra em processo de sucessão secundária.

Na distribuição das espécies em grupos sucessionais
(Fig. 1), a predominância de espécies secundárias iniciais e tardias mostra o avanço da sucessão neste fragmento da APA da Serra da Capoeira Grande. Porém, quando se avalia os grupos sucessionais em relação ao número de indivíduos, nota-se clara predominância de indivíduos pertencentes ao grupo das secundárias iniciais, o que indica que a área ainda não alcançou seu 
Tabela 3. Famílias com maior número de espécies (em percentagem) encontradas em diferentes trechos de Floresta Atlântica no Estado do Rio de Janeiro, de acordo com o estudo atual e os de outros autores.

\begin{tabular}{lllll}
\hline Rodrigues (1995) & Farág(1999) & Kurtz \& Araujo (2000) & Silva \& Nascimento (2001) & Este estudo \\
\hline Myrtaceae-12,2 & Myrtaceae-22 & Myrtaceae-19,6 & Leguminosae-20,5 & Leguminosae-19,1 \\
Rubiaceae-12,2 & Leguminosae-19 & Sapotaceae-10,1 & Myrtaceae-9,6 & Myrtaceae-8,8 \\
Leguminosae-8,5 & Euphorbiaceae-8 & Lauraceae-10,1 & Euphorbiaceae-7,2 & Euphorbiaceae-7,4 \\
Meliaceae-5,3 & Nyctaginaceae-4 & Rubiaceae-6,5 & Lecythidaceae-6,0 & Bignoniaceae-4,4 \\
Lauraceae-4,8 & Sapotaceae-4 & Meliaceae-4,3 & Rutaceae-4,8 & Bombacaceae-4,4 \\
\hline
\end{tabular}

desenvolvimento total, ou que existe algum fator que está impedindo o pleno desenvolvimento desta floresta. Entre os fatores que podem estar retardando o avanço sucessional neste trecho, pode-se citar a ocorrência de fogo e o corte seletivo de algumas espécies, eventos que acontecem até os dias de hoje, principalmente por este fragmento estar inserido em área residencial, fato este percebido também pelo grande número de espécies frutíferas exóticas que ocorrem no local.

Comparação entre fragmentos - A localização dos fragmentos florestais utilizados para comparação entre diversos remanescentes no Estado do Rio de Janeiro está apresentada na Figura 2, e na Tabela 4, são apresentadas as características ambientais, vegetacionais e o método de amostragem utilizado em cada trabalho.

Observa-se que o índice de similaridade só foi superior a $25 \%$ duas vezes (Fig. 3), destacando a baixa semelhança florística entre as áreas (Mueller-Dombois \& Ellenberg 1974). A heterogeneidade florística entre florestas no Domínio da Floresta Atlântica tem sido apontada em vários estudos e vem sendo atribuída a uma série de fatores, como o número elevado de espécies representadas por um ou poucos indivíduos (Pagano et al. 1995), baixa constância (distribuição em manchas)

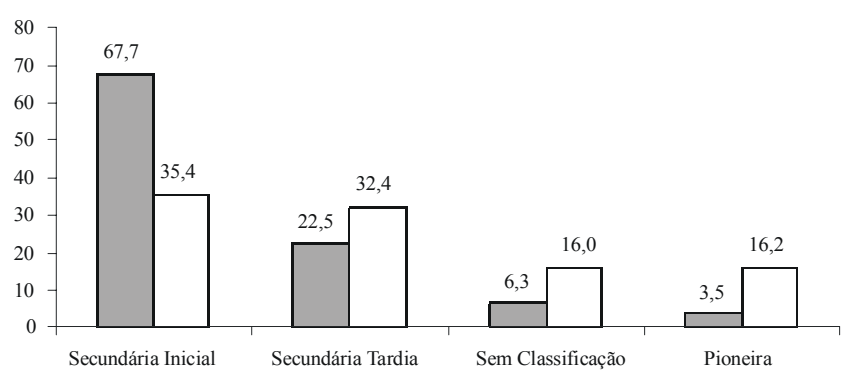

Figura 1. Distribuição das espécies arbóreas e dos indivíduos encontrados na Área de Proteção Ambiental da Serra da Capoeira Grande, Rio de Janeiro, RJ, em categorias ecológicas sucessionais. $\square$ Número de indivíduos; $\square$ Número de espécies.

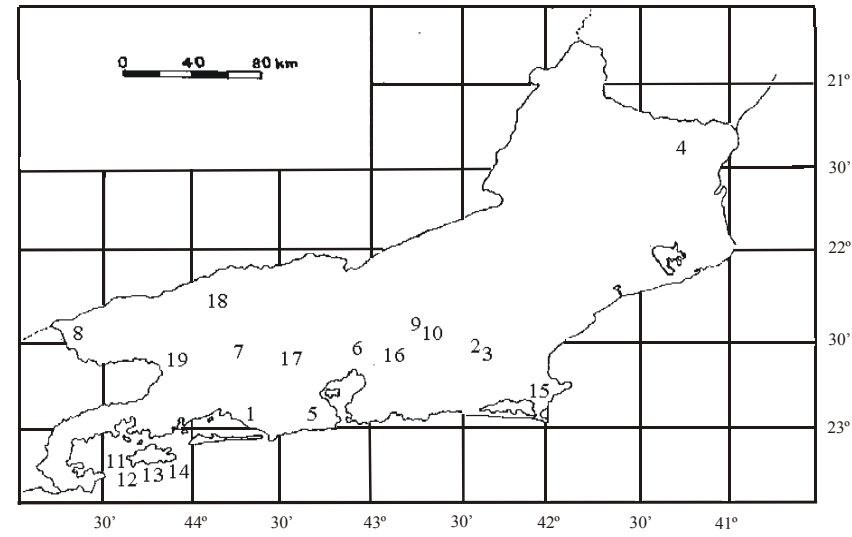

Figura 2. Mapa de localização dos fragmentos de Mata Atlântica do Estado do Rio de Janeiro utilizados na análise de similaridade florística. Os números correspondem às áreas apresentadas na Tabela 4.

de muitas espécies, com localidades distintas apresentando diferentes espécies (Scudeller et al. 2001), mosaicos sucessionais gerados pela ocupação de clareiras (Tabarelli \& Mantovani 1997) e variações climáticas, altitudinais e edáficas (Torres et al. 1997; Oliveira-Filho \& Fontes 2000).

Apesar da heterogeneidade florística entre localidades, cinco grupos podem ser identificados na análise de agrupamento (Fig. 3). Primeiramente, o grupo um difere dos demais com um baixo índice de Jaccard (separação indicada pelas letras a1 e a2). Em seguida, os grupos dois, três e quatro são separados do grupo cinco (separação indicada pelas letras b1 e b2). Posteriormente, as duas áreas localizadas na Reserva Ecológica de Macaé de Cima (grupo quatro) são separadas dos grupos dois e três (separação indicada pelas letras c1 e c2) e, finalmente, há a separação dos grupos dois e três indicada pelas letras $\mathrm{d} 1 \mathrm{e} \mathrm{d} 2$.

O primeiro grupo (a2), na parte direita do dendrograma é formado pela APA da Serra da Capoeira Grande (1), pelo trecho do município de Búzios (15), onde também foi encontrado pau-brasil, e a Mata do Carvão, no norte fluminense (4). Essas três áreas têm 
Tabela 4. Características ambientais, vegetacionais e métodos de amostragem das 19 áreas de Floresta Atlântica do Estado do Rio de Janeiro utilizadas para a determinação da similaridade florística: número de espécies encontradas (spp); porcentagem de espécies raras (\% spp); índice de diversidade (H'); método utilizado e esforço amostral (MET); critério de inclusão (CRIT): DAP e PAP em centímetros e H em metros; altitude da área em metros (ALT); pluviosidade em mm (PLUV).

\begin{tabular}{|c|c|c|c|c|c|c|c|c|c|}
\hline $\mathrm{N}$ & spp & $\%$ spp. & . $\mathrm{H}^{\prime}$ & MET & CRIT & ALT & PLUV & Área estudada & Fonte \\
\hline 1 & 68 & 37,2 & 2,42 & Quadrante200 pontos & $\mathrm{PAP} \geq 15$ & 60 a 150 & $1.027,2$ & APA da Capoeira Grande & Este trabalho \\
\hline 2 & 97 & 35,1 & 3,99 & Parcela $0,25 \mathrm{ha}$ & $\mathrm{DAP} \geq 2,5$ & 0 a 200 & $2.087 \mathrm{R}$ & REBIO Poço das Antas (20 anos) & Neves (2001) \\
\hline 3 & 169 & 50,1 & 4,55 & Parcela $0,25 \mathrm{ha}$ & $\mathrm{DAP} \geq 2,5$ & 0 a 200 & $2.087 \mathrm{R}$ & REBIO Poço das Antas (40 anos) & Neves (2001) \\
\hline 4 & 83 & - & 3,21 & Parcela 1 ha & $\mathrm{DAP} \geq 10$ & - & 1.023 & Mata do Carvão & $\begin{array}{l}\text { Silva \& Nascimento } \\
\text { (2001) }\end{array}$ \\
\hline 5 & 139 & 38,1 & - & Parcela 0,25 ha & $\mathrm{DAP} \geq 2,5$ & "716" & - & Vertente N (Tijuca) & Oliveira et al. (1995) \\
\hline & 155 & 45,2 & & & & & & Vertente S (Tijuca) & \\
\hline 6 & 90 & 43,3 & - & Parcela 0,2 ha & $\mathrm{H} \geq 2$ & 0 a 40 & 2.050 & Município de Magé & Guedes (1988) \\
\hline 7 & 192 & & & $\begin{array}{l}\text { Lista Florística } \\
\text { (todas as árvores) }\end{array}$ & & 400 & 1.368 a 2.067 & Ribeirão das Lages & Peixoto et al. (1995) \\
\hline 8 & 150 & 48,0 & 3,86 & Parcela 1ha & $\mathrm{DAP} \geq 10$ & Até "2.787" & $" 1.765,8$ & P.N. Itatiaia & Guedes-Bruni (1998) \\
\hline 9 & 157 & 32,5 & 3,66 & Parcela 1ha & $\mathrm{DAP} \geq 5$ & 1.0001 & 1.500 a 2.000 & $\begin{array}{l}\text { R.E. Macaé de Cima } \\
\text { (área preservada) }\end{array}$ & Pessoa et al. (1997) \\
\hline 10 & 189 & 34,4 & 4,05 & Parcela1ha & $\mathrm{DAP} \geq 5$ & 1.1001 . & 1.500 a 2.000 & $\begin{array}{l}\text { R.E. Macaé de Cima } \\
\text { (área perturbada) }\end{array}$ & $\begin{array}{l}\text { Guedes-Bruni et al. } \\
\text { (1997) }\end{array}$ \\
\hline 11 & 26 & 3,8 & 2,51 & Parcela $0,26 \mathrm{ha}$ & $\begin{aligned} \mathrm{DAP} & \geq 2,54 \\
\mathrm{H} & \geq 2\end{aligned}$ & 80 a 280 & $1.975,0$ & P. E. Ilha Grande (5 anos) & Oliveira (1999) \\
\hline 12 & 70 & 32,8 & 3,33 & Parcela $0,26 \mathrm{ha}$ & $\begin{array}{c}\text { DAP } \geq 2,54 \\
\mathrm{H} \geq 2\end{array}$ & 80 a 280 & $1.975,0$ & P. E. Ilha Grande (25 anos) & Oliveira (1999) \\
\hline 13 & 63 & 41,3 & 3,10 & Parcela $0,26 \mathrm{ha}$ & $\begin{array}{c}\mathrm{DAP} \geq 2,54 \\
\mathrm{H}^{3} 2\end{array}$ & 80 a 280 & $1.975,0$ & P. E. Ilha Grande (50 anos) & Oliveira (1999) \\
\hline 14 & 134 & 42,5 & 4,28 & Parcela $0,26 \mathrm{ha}$ & $\begin{aligned} \mathrm{DAP} & \geq 2,54 \\
\mathrm{H} & \geq 2\end{aligned}$ & 80 a 280 & $1.975,0$ & P. E. Ilha Grande (área clímax) & Oliveira (1999) \\
\hline 15 & 124 & 17,7 & 4,00 & Parcela 0,5 ha & $\mathrm{DAP} \geq 5$ & $\approx 0$ & 822 & Município de Búzios & Farág (1999) \\
\hline 16 & 138 & 44,9 & 4,20 & Quadrante 150 pontos & $\mathrm{DAP} \geq 5$ & $" 60$ a $1.350 "$ & $" 2.558,4$ & E.E.E. de Paraíso & Kurtz \& Araújo (2000) \\
\hline 17 & 189 & 46,0 & 4,36 & Quadrante 200 pontos & $\mathrm{DAP} \geq 2,54$ & 125 & $2.099,3$ & REBIO Tinguá & Rodrigues (1996) \\
\hline 18 & 85 & - & - & Parcela 0,3 ha & $\mathrm{DAP} \geq 5$ & 364 & $1.258,2$ & C.E. Santa Mônica & Spolidoro (2001) \\
\hline 19 & 184 & 47,3 & 3,66 & Parcela 0,3 ha & $\mathrm{DAP} \geq 2,5$ & 360 & - & ARIE Floresta da Cicuta & Souza (2002) \\
\hline
\end{tabular}

em comum o fato de estarem próximas ao mar (Fig. 2), terem baixa pluviosidade (aproximadamente $1.000 \mathrm{~mm}$ anuais) e de estarem localizadas em baixadas (Tab. 4). Nestas áreas ocorrem espécies características de planícies e pequenas elevações costeiras que não foram encontradas nos outros levantamentos. Foram três as espécies encontradas apenas nestas áreas: Acosmium lentiscifolium (Leguminosae), Machaerium incorruptible (Leguminosae) e Metternichia princeps (Solanaceae). Essas espécies são características de ambientes costeiros mais secos e podem ser usadas como indicadoras destes locais. Acosmium lentiscifolium e Machaerium incorruptible estão na lista de espécies ameaçadas no município do Rio de Janeiro (Di Maio \& Silva 2000). Esse fato pode mostrar que não só a exploração seletiva do pau-brasil o levou a estar ameaçado de extinção, mas a destruição de hábitats preferenciais tem colocado em risco várias espécies que ocorrem nesses ambientes e que podem ser consideradas espécies companheiras. Além disso, os dados sobre endemismo dessas leguminosas mostram a necessidade de preservação de seu hábitat, que está sob forte pressão antrópica e exige medidas urgentes para sua preservação. Lima (2000) destacou que a maior diversidade de leguminosas no Rio de Janeiro ocorre em elevações situadas entre 50 e 500 m.s.m. e realçou a importância da preservação dos trechos de florestas nessas cotas altitudinais.

Metternichia princeps, espécie com maior número de indivíduos na APA da Serra da Capoeira Grande, também só foi encontrada entre os trechos estudados, em Búzios e na Mata do Carvão. Espécies características de florestas submetidas a certa estacionalidade de chuvas estão presentes na região litorânea, nos tabuleiros costeiros do sul do Estado do Espírito Santo e do norte do Estado do Rio de Janeiro até a região de Cabo Frio (RADAMBRASIL 1983; Oliveira-Filho \& Fontes 2000). O presente trabalho vem mostrar que estas espécies características de florestas estacionais estão presentes nas áreas de planície costeira do Estado do Rio de Janeiro, chegando a alcançar regiões mais ao sul nesse Estado, onde as 


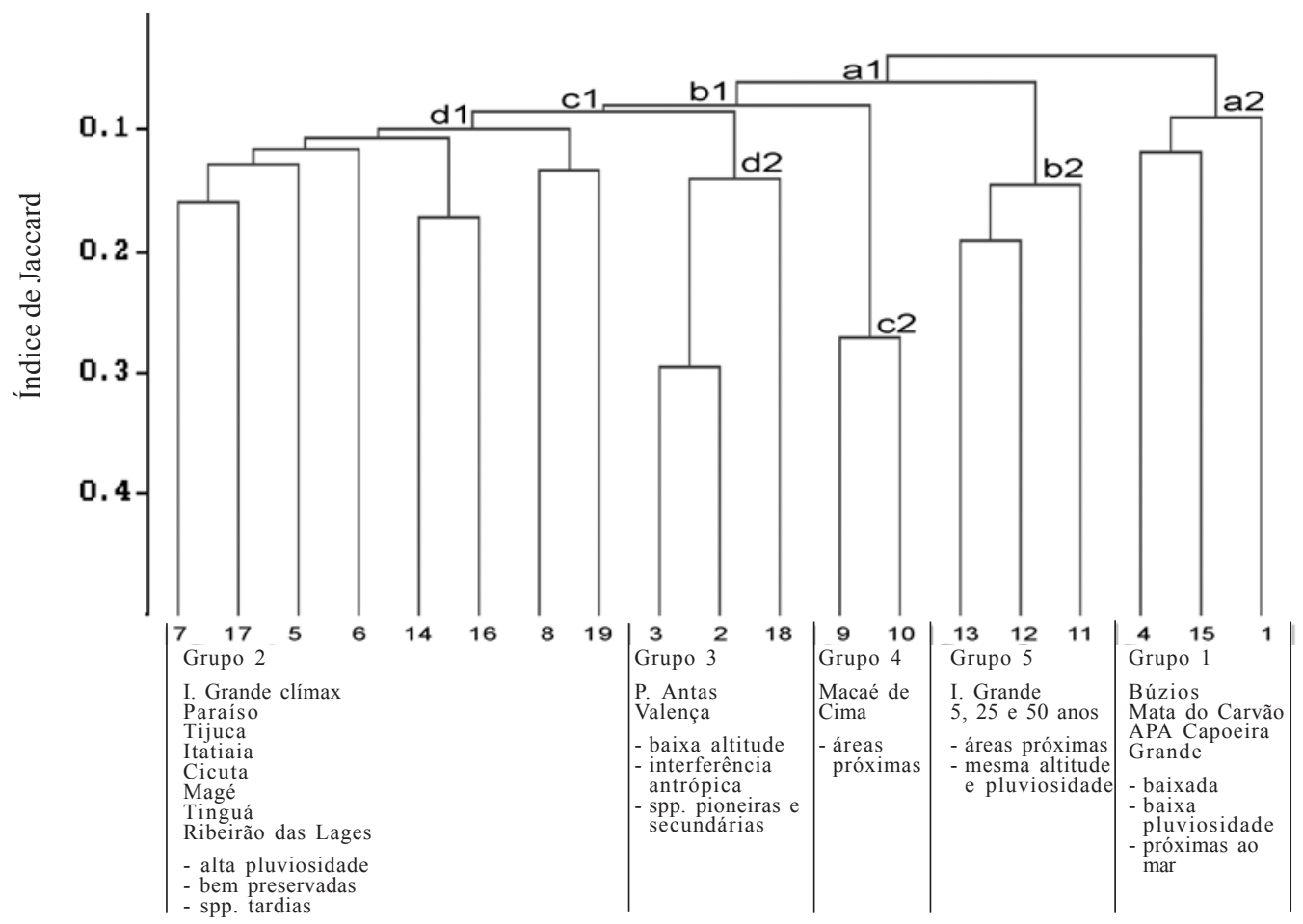

Figura 3. Dendrograma de similaridade florística entre trechos de Floresta Atlântica no Estado do Rio de Janeiro. Os números correspondem às áreas apresentadas na Tabela 4.

características locais, principalmente a baixa pluviosidade e a existência de período mais seco, permitem sua presença.

O segundo grupo (d1), na parte esquerda do gráfico, formado pelos trechos da Ilha Grande clímax (14), Mata do Paraíso (16), Floresta da Tijuca (5), Itatiaia (8), Floresta da Cicuta (19), Magé (6), Tinguá (17) e Ribeirão das Lages (7), apresenta como característica comum a alta pluviosidade. Essas áreas estão distribuídas desde o litoral até o interior do Estado, em altitudes que variam de 80 a mais de 2.000 m.s.m. (Tab. 4) Acredita-se que a formação desse grupo se deva ao bom estado de preservação dos trechos estudados. Essas áreas não apresentam histórico recente de alteração antrópica e seriam, portanto, florestas maduras, estando representadas principalmente por espécies secundárias tardias, como Cabralea canjerana (Meliaceae), Guarea macrophylla (Meliaceae), Licania kunthiana (Chrysobalanaceae) e Cariniana estrellensis (Lecythidaceae).

O terceiro grupo (d2), no centro do gráfico, formado pelos trechos de Poço das Antas (2 e 3) e Valença (18), tem algumas características em comum, o que provavelmente fez com que as espécies que ocorrem nessas áreas sejam semelhantes. Primeiramente, pode-se citar a baixa altitude, pois as duas áreas estudadas estão em altitudes que alcançam no máximo 400m (Tab. 4). Porém, a semelhança florística entre essas áreas deve-se, principalmente, ao histórico da ocupação humana. Todas elas foram submetidas a impacto antrópico muito semelhante, devendo, esse grupo, estar sendo influenciado pelo elevado número de espécies pioneiras e secundárias iniciais como Cybistax antisyphilitica (Bignoniaceae), Sparattosperma leucanthum (Bignoniaceae), Nectandra membranacea (Lauraceae) e Apuleia leiocarpa (Leguminosae).

As duas áreas de Macaé de Cima (9 e 10), uma perturbada e outra preservada, formaram um grupo isolado (grupo c2), provavelmente por serem trechos muito próximos; possivelmente o perturbado deveria ficar no segundo grupo e o preservado, no terceiro. Porém, a semelhança florística entre estas duas áreas não permitiu que fossem separadas, ficando assim numa posição intermediária entre os dois grandes grupos.

O quinto grupo (b2), formado pelos trechos da Ilha Grande, de 5, 25 e 50 anos (11, 12 e 13), ficou isolado dos demais pela semelhança de diversas características entre as áreas. Os três trechos estão em áreas de cultivo abandonado, têm a mesma altitude e pluviosidade e são bastante próximos entre si. Assim, apesar de estarem 
em diferentes estádios de sucessão, apresentam muitas espécies em comum, como Tabernaemontana laeta (Apocynaceae), Ilex integerrima (Aquifoliaceae) e Lamanonia ternata (Cunoniaceae).

A floresta estudada na APA da Serra da Capoeira Grande representa importante remanescente de Floresta Atlântica, com a presença de duas espécies arbóreas que estão na lista oficial da flora brasileira ameaçada de extinção: Caesalpinia echinata, Cariniana ianeirensis; e outras duas que estão na lista das espécies ameaçadas de extinção no município do Rio de Janeiro: Acosmium lentiscifolium e Machaerium incorruptible, devendo ser utilizada como fonte de germoplasma para a produção de mudas dessas e de outras espécies. Recomenda-se como estratégia para evitar a extinção local dessas espécies, o plantio de enriquecimento neste fragmento e em áreas em processo de sucessão daqueles fragmentos que apresentaram maior semelhança florística e ambiental, que são os municípios de Búzios e de São Francisco de Itabapoana.

\section{Agradecimentos}

Os Autores agradecem aos professores e pesquisadores que auxiliaram na identificação do material botânico: Haroldo Cavalcante de Lima, Ariane Luna Peixoto, Graziela Maciel Barroso, Ronaldo Marquete, Luci de Senna Vale, Lucia d'Avila Freire de Carvalho, Alexandre Quinet, Elsie Franklin Guimarães, Maria Verônica Leite Pereira-Moura, Sebastião José da Silva Neto e Scott Mori; à Coordenadoria de Aperfeiçoamento de Pessoal de Nível Superior CAPES, pela concessão da Bolsa de Mestrado.

\section{Referências bibliográficas}

Alvarez V., V. H.; Novais, R. F.; Barros, N. F.; Cantarutti, R. B. \& Lopes, A. S. 1999. Interpretação dos resultados das análises de solo. In: A. C. Ribeiro, P. T. G. Guimarães \& V. H. Alvarez V. (eds.). Recomendações para uso de corretivos e fertilizantes em Minas Gerais. CFSEMG, Viçosa.

CIDE. 2000. Índice de qualidade dos municípios - verde. Centro de Informações e Dados do Rio de Janeiro, Rio de Janeiro.

Consorcio Mata Atlântica. 1992. Reserva da Biosfera da Mata Atlântica - Plano de Ação. Volume 1: Referências Básicas. UNICAMP, Campinas.

Cottam G. \& Curtis, J. T. 1956. The use of distance measures in phytosociological sampling. Ecology 37(3): 451-460.

Dean, W. 1995. A ferro e fogo: a história e a devastação da Mata Atlântica brasileira. Companhia das Letras, São Paulo.
Di Maio, F.R. \& Silva, M.B.R. 2000. Espécies ameaçadas de extinção no município do Rio de Janeiro. Secretaria Municipal do Meio Ambiente, Rio de Janeiro.

EMBRAPA. 1979. Manual de métodos de análise de solo. EMBRAPA-SNLCS, Rio de Janeiro.

Farág, P. R. C. 1999. Estrutura do estrato arbóreo de mata litorânea semicaducifólia sobre solo arenoso no município de Búzios, RJ. Dissertação de Mestrado. Museu Nacional da Universidade Federal do Rio de Janeiro, Rio de Janeiro.

Gandolfi, S.; Leitão-Filho, H. F. \& Bezerra, C. L. F. 1995. Levantamento florístico e caráter sucessional das espécies arbustivo-arbóreas de uma floresta mesófila semidecídua no município de Guarulhos, SP. Revista Brasileira de Biologia 55(4): 753-767.

Guedes, R. R. 1988. Composição florística e estrutura de um trecho de mata perturbada de baixada no município de Magé, Rio de Janeiro. Arquivos do Jardim Botânico do Rio de Janeiro 39: 155-200.

Guedes-Bruni, R. R.; Pessoa, S. V. A. \& Kurtz, B. C. 1997. Florística e estrutura do componente arbustivo-arbóreo de um trecho preservado de floresta montana na Reserva Ecológica de Macaé de Cima. Pp. 127-145. In: H.C. Lima \& R. R. Guedes-Bruni (eds.). Serra de Macaé de Cima: diversidade, florística e conservação em Mata Atlântica. Jardim Botânico do Rio de Janeiro, Rio de Janeiro.

Guedes-Bruni, R. R. 1998. Composição, estrutura e similaridade $\mathrm{f}$ de dossel em seis unidades fisionômicas de Mata Atlântica no Rio de Janeiro. Tese de Doutorado. Universidade de São Paulo, São Paulo.

IUCN. 1986. Plants in danger. What do we Know? Cambridge.

Kurtz, B. C. \& Araújo, D. S. D. 2000. Composição florística e estrutura do componente arbóreo de um trecho de Mata Atlântica na Estação Ecológica Estadual de Paraíso, Cachoeira de Macacu, Rio de Janeiro, Brasil. Rodriguésia 51: 69-112.

Leitão-Filho, H. F. 1993. (Org.) Ecologia da Mata Atlântica em Cubatão (SP). UNICAMP, Campinas.

Lima H. C. 2000. Leguminosas arbóreas da Mata Atlântica. Tese de Doutorado. Universidade Federal do Rio de Janeiro, Rio de Janeiro.

Lorenzi, H. 1998. Árvores brasileiras: manual de identificação e cultivo de plantas arbóreas nativas do Brasil. V.2. 2.ed. Ed. Plantarun, Nova Odessa.

Lorenzi, H. 2000. Árvores brasileiras: manual de identificação e cultivo de plantas arbóreas nativas do Brasil. V.1. 3.ed. Ed. Plantarun, Nova Odessa.

Martins, F. R. 1993. Estrutura de uma floresta mesófila. UNICAMP, Campinas.

Martins, S.V.; Coutinho, M.P. \& Marangon, L.C. 2002. Composição florística e estrutura de uma floresta secundária no município de Cruzeiro, SP. Revista Árvore, 26(1): 35-41.

Menezes, L. F. T. \& Araújo, D. S. D. 1999. Estrutura de duas formações vegetais do cordão externo da Restinga da Marambaia, RJ. Acta Botanica Brasilica 13(2): 223-235.

Mori, S. A.; Boom, B. M.; Carvalho, A. M. \& Santos, T. S. 1983. Ecological importance of Myrtaceae in Eastern Brazilian wet forest. Biotropica 15: 68-70. 
Mueller-Dombois, D. \& Ellenberg, H. 1974. Aims and methods of vegetation ecology. John Wiley \& Sons, New York.

Neves, G. M. S. 2001. Florística e estrutura da comunidade arbustivo-arbórea em dois remanescentes de Floresta Atlântica secundária - Reserva Biológica de Poço das Antas, Silva Jardim, RJ. Dissertação de Mestrado. Museu Nacional, Universidade Federal do Rio de Janeiro, Rio de Janeiro.

Oliveira, R. R.; Zaú, A. S.; Lima, D. F.; Silva, M. B. R.; Vianna, M. C.; Sodré, D. O. \& Sampaio, P. D. 1995. Significado ecológico da orientação de encostas no maciço da Tijuca, Rio de Janeiro. Oecologia Brasiliensis 1: 523-541.

Oliveira, R. R. 1999. O rastro do homem na floresta: Sustentabilidade e funcionalidade da Mata Atlântica sob o manejo caiçara. Tese de Doutorado. Universidade Federal do Rio de Janeiro, Rio de Janeiro.

Oliveira-Filho, A. T. \& Fontes, M. A. L. 2000. Patterns of floristic differentation among Atlantic Forests in Southeastern Brazil and the influence of climate. Biotropica 32(4b): 793-810.

Pagano, S. N.; Leitão-Filho, H. F. \& Cavassan, O. 1995. Variação temporal da composição florística e estrutura fitossociológica de uma floresta mesófila semidecídua Rio Claro, SP. Revista Brasileira de Biologia 55(2): 241-258.

Peixoto, A. L. \& Gentry, A. 1990. Diversidade e composição florística da mata de tabuleiro na Reserva Florestal de Linhares (Espirito Santo, Brasil). Revista Brasileira de Botânica 13: 19-25.

Peixoto, A.L. 1992. Vegetação da Costa Atlântica. Pp. 3341. In: S. Monteiro \& L. Kas (eds.). Floresta Atlântica. Edições Alumbramento/ Livroarte Editora, Rio de Janeiro.

Peixoto, A. L.; Rosa, M. M. T.; Barbosa, M. R. V. \& Rodrigues. H. C. 1995. Composição florística da área em torno da represa de Ribeirão das Lages, Rio de Janeiro, Brasil. Revista da Universidade Rural, Série Ciências da Vida 17(1): 51-74.

Pessoa, S. V. A.; Guedes-Bruni, R. R. \& Kurtz, B. C. 1997. Composição florística e estrutura do componente arbustivo-arbóreo de um trecho secundário de floresta montana na Reserva Ecológica de Macaé de Cima. Pp.148167. In: H. C. Lima \& R. R. Guedes-Bruni (eds.). Serra de Macaé de Cima: diversidae florística e conservação em Mata Atlântica. Jardim Botânico do Rio de Janeiro, Rio de Janeiro.
RADAMBRASIL. 1983. Folhas SF. 23/24 Rio de Janeiro/ Vitória; geologia, geomorfologia, pedologia, vegetação e uso potencial da terra. Projeto RADAMBRASIL, Rio de Janeiro.

Rodrigues, H. C. 1996. Composição florística e fitossociológica de um trecho de Mata Atlântica na Reserva Biológica do Tinguá, Nova Iguaçu, Rio de Janeiro. Dissertação de Mestrado. Museu Nacional, Universidade Federal do Rio de Janeiro, Rio de Janeiro

Santana, C. A. A. 2000. Estrutura e dinâmica de florestas secundárias de encosta no município do Rio de Janeiro. Dissertação de Mestrado. Universidade Federal Rural do Rio de Janeiro, Rio de Janeiro.

Scudeller, V. V.; Martins F. R. \& Shepherd, G. J. 2001. Distribution and abundance of arboreal species in the Atlantic ombrophilous dense forest in Southeastern Brazil. Plant Ecology 152: 185-199.

Shepherd, G.J. 1996. FITOPAC 1: Manual do usuário. UNICAMP, Campinas.

Silva, G. C. \& Nascimento M. T. 2001. Fitossociologia de um remanescente de mata sobre tabuleiros no norte do estado do Rio de Janeiro (Mata do Carvão). Revista Brasileira de Botânica 24(1): 51-62.

Souza, G. R. 2002. Florística do estrato arbustivo-arbóreo em um trecho de Floresta Atlântica, no médio Paraíba do Sul, município de Volta Redonda, Rio de Janeiro. Dissertação de Mestrado. Universidade Federal Rural do Rio de Janeiro, Rio de Janeiro.

Spolidoro, M. L. C. V. 2001. Composição e estrutura em um trecho de floresta no médio Paraíba do Sul, RJ. Dissertação de Mestrado. Universidade Federal Rural do Rio de Janeiro, Rio de Janeiro.

Tabarelli, M. 1997. A regeneração da Floresta Atlântica montana. Tese de Doutorado. Universidade de São Paulo, São Paulo.

Tabarelli, M. \& Mantovani, W. 1997.Colonização de clareiras naturais na floresta atlântica no sudeste do Brasil. Revista Brasileira de Botânica 20(1): 57-66.

Torres, R. B.; Martins, F. R.; Kinoshita, L. S. 1997. Climate, soil and tree flora relationships in forest in the State of São Paulo, souheastern Brasil. Revista Brasileira de Botânica 20(1): 41-49.

Valentim, J. L. 2000. Ecologia numérica: Uma introdução à análise multivariada de dados. Editora Interciências, Rio de Janeiro. 